\title{
QRS Voltage-Duration Product in the Identification of Left Ventricular Hypertrophy in Spontaneously Hypertensive Rats
}

\author{
Ljuba Bacharova, Jan Kyselovic, Jan Klimas \\ Bratislava - Slovak Republic
}

\begin{abstract}
Objective - Evaluation of the performance of the $Q R S$ voltage-duration product (VDP) for detection of left ventricular hypertrophy $(\mathrm{LVH})$ in spontaneously hypertensive rats (SHR).

Methods - Orthogonal electrocardiograms (ECG) were recorded in male SHR at the age of 12 and 20 weeks, when systolic blood pressure $(S B P)$ reached the average values of $165 \pm 3 \mathrm{mmHg}$ and $195 \pm 12 \mathrm{mmHg}$, respectively. Age- and sex-matched normotensive Wistar Kyoto (WKY) rats were used as controls. VDP was calculated as a product of maximum $Q R S$ spatial vector magnitude and $Q R S$ duration. Left ventricular mass (LVM) was weighed after rats were sacrificed.
\end{abstract}

Results - LVM in SHR at 12 and 20 weeks of age (0.86 $\pm 0.05 \mathrm{~g}$ and $1.05 \pm 0.07 \mathrm{~g}$, respectively) was significantly higher as compared with that in WKY $(0.65 \pm 0.07 \mathrm{~g}$ and $0.70 \pm 0.02 \mathrm{~g}$ ). The increase in LVM closely correlated with the sBP increase. VDP did not reflect the increase in LVM in SHR. $V D P$ was lower in SHR as compared with that in WKY, and the difference was significant at the age of 20 weeks $(18.2 \mathrm{mVms}$ compared with $10.7 \mathrm{mVms}, p<0.01)$. On the contrary, a significant increase in the VDP was observed in the control WKY at the age of 20 weeks without changes in LVM. The changes in $V D P$ were influenced mainly by the changes in QRSmax.

Conclusion - LVM was not the major determinant of $Q R S$ voltage changes and consequently of the VDP. These data point to the importance of the nonspatial determinants of the recorded $Q R S$ voltage in terms of the solid angle theory.

Keywords: left ventricular hypertrophy, electrocardiography, QRS voltage-duration product, spontaneously hypertensive rat

International Laser Center, Ilkovicova 3, 812 19, Slovak Republic Pharmaceutical Faculty Comenius University, Kalinciakova 8, 83232 Bratislava, Slovak Republic Mailing address: Ljuba Bacharova, MD, PhD, MBA - International Laser Center -Ilkovicova 3 - 81219 Bratislava - Slovak Republic - E-mail: bacharova@ilc.sk Received for publication in October 23, 2001

Accepted in November 28, 2001
Electrocardiographic signs of left ventricular hypertrophy (ECG-LVH) are a powerful independent predictors of cardiovascular morbidity and mortality. Based on 36 years of follow-up in the Framingham study, ECG-LVH was found to increase the risk of coronary events about 3-fold to 5-fold, strokes 6-fold, and heart failure about 14 -fold ${ }^{1}$. The electrocardiographic criteria based on the increased QRS voltage the voltage criteria - are highly specific for LVH detection. ECG is an easily applicable, clinically relevant method that, together with its low cost, represents additional advantages. On the other hand, its main limitation is the high number of false negatives resulting in poor sensitivity of the voltage criteria. Therefore, a continuous effort is devoted to improving the performance of ECG criteria in LVH detection.

The simple product of QRS duration and voltage, as an approximation of the time-duration area under the QRS complex, has been shown to enhance the sensitivity of the ECG identification of left ventricular hypertrophy as defined at autopsy ${ }^{2}$, as well as of increased left ventricular mass detected by echocardiography in living subjects ${ }^{3}$. The Cornell product has been reported to provide low variability of performance between definitions of hypertrophy in terms of the different upper normal limits used ${ }^{4}$. Use of voltage-duration products mitigates the negative impact of increased BMI on the prevalence of $\mathrm{LVH}$, and it has been suggested that the voltage-duration products may be the most accurate conventional ECG method for detecting anatomic LVH, independent of body habitus ${ }^{5,6}$.

Similar results have also been reported by others. The Cornell voltage duration product exhibited the greatest sensitivity for both sexes (39\% in men and $51 \%$ in women at $95 \%$ specificity). The Cornell voltage duration product adjusted for body mass index and age offers significant improvement for the detection of echocardiographically determined LVH in all but lean men ${ }^{7}$.

ECG evaluation based either on sex-specific orthogonal time-voltage criteria or on the combined standard 12lead criteria of Sokolow-Lyon or the Cornell product demonstrates the best overall diagnostic accuracy. With orthogonal criteria, the sensitivity is $81 \%$ in women and $71 \%$ in 
men. At a matched $98 \%$ specificity (the corresponding overall sensitivity of the combined Cornell product or SokolowLyon criteria) is reported to be $68 \%$ at a specificity of $96.6 \%$.

The spontaneously hypertensive rat (SHR) is regarded as a reliable model of human essential hypertension ${ }^{8,9}$. SHRs have, within each colony, uniform polygenic disposition and excitatory factors, which produce uniform changes in the indirect and direct effects on the cardiovascular system. This model also provides good control of variables like age and duration of hypertension. The lack of interindividual variation is one of the major advantages of the SHR ${ }^{10}$. Furthermore, the SHR is used as a realistic model of left ventricular hypertrophy, because of the gradual onset of systemic pressure overload that occurs. LVH in SHR develops naturally without any invasive, chemical, or pharmacological interventions, and proceeds chronically. Although in clinical studies the inconsistency of results has necessitated consideration of many extracardial factors, such as genetic variations, the unknown duration of hypertension, interindividual variability due to race, sex, or variability due to body habitus, in the experimental model of SHR these variables are well controlled.

The aim of this study was to assess the performance of the voltage-duration product as a parameter of $\mathrm{LVH}$ detection in the experimental model of spontaneously hypertensive rats in the early stage of hypertension and LVH development.

\section{Methods}

Spontaneously hypertensive rats were used as an experimental model of left ventricular hypertrophy due to hypertension. Two groups of male SHR at the age of 12 and 20 weeks were examined. Age- and sex-matched Wistar Kyoto rats (WKY) were used as controls. Each group consisted of 7 rats randomly selected from a large population of SHR and of control animals, respectively (both animal groups from Anlab, Prague, Czech Republic).

Arterial systolic blood pressure (sBP) was measured by the tail-cuff method in conscious animals prewarmed to $35^{\circ} \mathrm{C}$ in thermostatic cages. Measurements were repeated several times, and 3 values after stabilization were averaged. At the age of 12 weeks, systolic blood pressure reached the average value of $165 \pm 3 \mathrm{mmHg}$, and then at the age of 20 weeks, the sBP increased to $195 \pm 12 \mathrm{mmHg}$. The sBP values in WKY control groups were within normal limits $(122 \pm 8 \mathrm{mmHg}$ and $130 \pm 4 \mathrm{mmHg}$, respectively).

Orthogonal electrocardiograms of the Frank lead system were recorded in thiopental anesthesia (Thiopental, VUAB, Czech Republic, $45 \mathrm{mg} / \mathrm{kg}$, i. p.) using the electrocardiograph 3NEK-1, GDR. Needle electrodes were used. The center of the chest electrodes was $1.5 \mathrm{~cm}$ from the xiphoid process on the sternum. The legs were fixed in the ventral position by elastic cords.

The maximum deflections of QRS complex were measured manually. All calculations were made on the average of 5 QRS complexes. These values were taken as X, Y,
$\mathrm{Z}$ components of the maximum spatial QRS vector magnitude (QRSmax). The QRSmax was calculated using the formula: $\mathrm{QRS} \max =\sqrt{\mathrm{x}^{2}+\mathrm{y}^{2}+\mathrm{z}^{2}}$

The QRS duration (QRSdur) was measured from the earliest onset of QRS complex to the lowest point of the $S$ wave in any of the orthogonal leads, in records taken with the paper speed of $200 \mathrm{~mm} / \mathrm{sec}$.

The voltage-duration product (VDP) was calculated as the product of QRSmax and QRS duration.

After ECG recording, the animals were sacrificed and the left ventricular mass was weighted. Two parameters were used as measures of anatomical left ventricular hypertrophy: left ventricular mass (LVM) and left ventricular mass to body weight ratio (LVM/BW).

Groups of animals were compared using the MannWhitney U-test. $\mathrm{P}<0.05$ was accepted as significant. The statistical analysis was performed using StatGraphics for Windows, version 5, microcomputer software package (Statistical Graphics Co. Rockville, USA, 1991).

This study was approved by the Ethics Committee of the Pharmaceutical Faculty of the Comenius University in Bratislava.

\section{Results}

The spontaneously hypertensive rats showed a progressive rise in systolic blood pressure. Arterial systolic blood pressure, left ventricular mass, and left ventricular mass to body weight ratio were significantly greater in SHR than in WKY, as shown in table I. Significant hypertrophy was found in SHR in terms of absolute left ventricular mass as well as of left ventricular mass relative to body weight. The LVM in SHR $(0.86 \pm 0.05 \mathrm{~g}$ and $1.05 \pm 0.07 \mathrm{~g}$, respectively $)$ were significantly higher as compared with that in to WKY $(0.65 \pm 0.07 \mathrm{~g}$ and $0.70 \pm 0.02 \mathrm{~g}, \mathrm{p}<0.001)$. The increase in LVM significantly correlated with the sBP increase both in WKY $(\mathrm{r}=0.6797, \mathrm{p}<0.01)$ and in SHR $(\mathrm{r}=0.7257, \mathrm{p}<0.01)$.

Values of the voltage-duration product and of its individual components, QRSmax and QRS duration, in WKY and SHR are presented in figure 1.

The voltage-duration product was lower in SHR as compared with that in WKY, and the difference was statis-

\begin{tabular}{|c|c|c|c|c|}
\hline \multicolumn{5}{|c|}{$\begin{array}{l}\text { Table I - Systolic blood pressure (sBP), left ventricular mass (LVM) and } \\
\text { left ventricular mass to body weight ratio (LVM/BW) in normotensive } \\
\text { WKY rats (WKY) and spontaneously hypertensive rats (SHR). }\end{array}$} \\
\hline & WKY & WKY & SHR & SHR \\
\hline & $12 \mathrm{w}$ & $20 \mathrm{w}$ & $12 \mathrm{w}$ & $20 \mathrm{w}$ \\
\hline $\mathrm{n}$ & 7 & 7 & 7 & 7 \\
\hline $\begin{array}{l}\mathrm{sBP} \\
{[\mathrm{mmHg}]}\end{array}$ & $124 \pm 8$ & $130 \pm 4$ & $165 \pm 3 * * *$ & $195 \pm 12 * * *$ \\
\hline LVM $[\mathrm{g}]$ & $0.65 \pm 0.07$ & $0.7 \pm 0.02$ & $0.86 \pm 0.05 * * *$ & $1.05 \pm 0.07 * * *$ \\
\hline $\begin{array}{l}\mathrm{LVM} / \mathrm{BW} \\
{[\mathrm{g} / \mathrm{kg}]}\end{array}$ & $2.13 \pm 0.15$ & $1.96 \pm 0.08$ & $2.96 \pm 0.15^{* * *}$ & $3.03 \pm 0.15 * * *$ \\
\hline \multicolumn{5}{|c|}{$\begin{array}{l}\text { Average values } \pm \text { SD are presented; statistically significant difference SHR } \\
\text { vs WKY: } * * *=p<0.001\end{array}$} \\
\hline
\end{tabular}




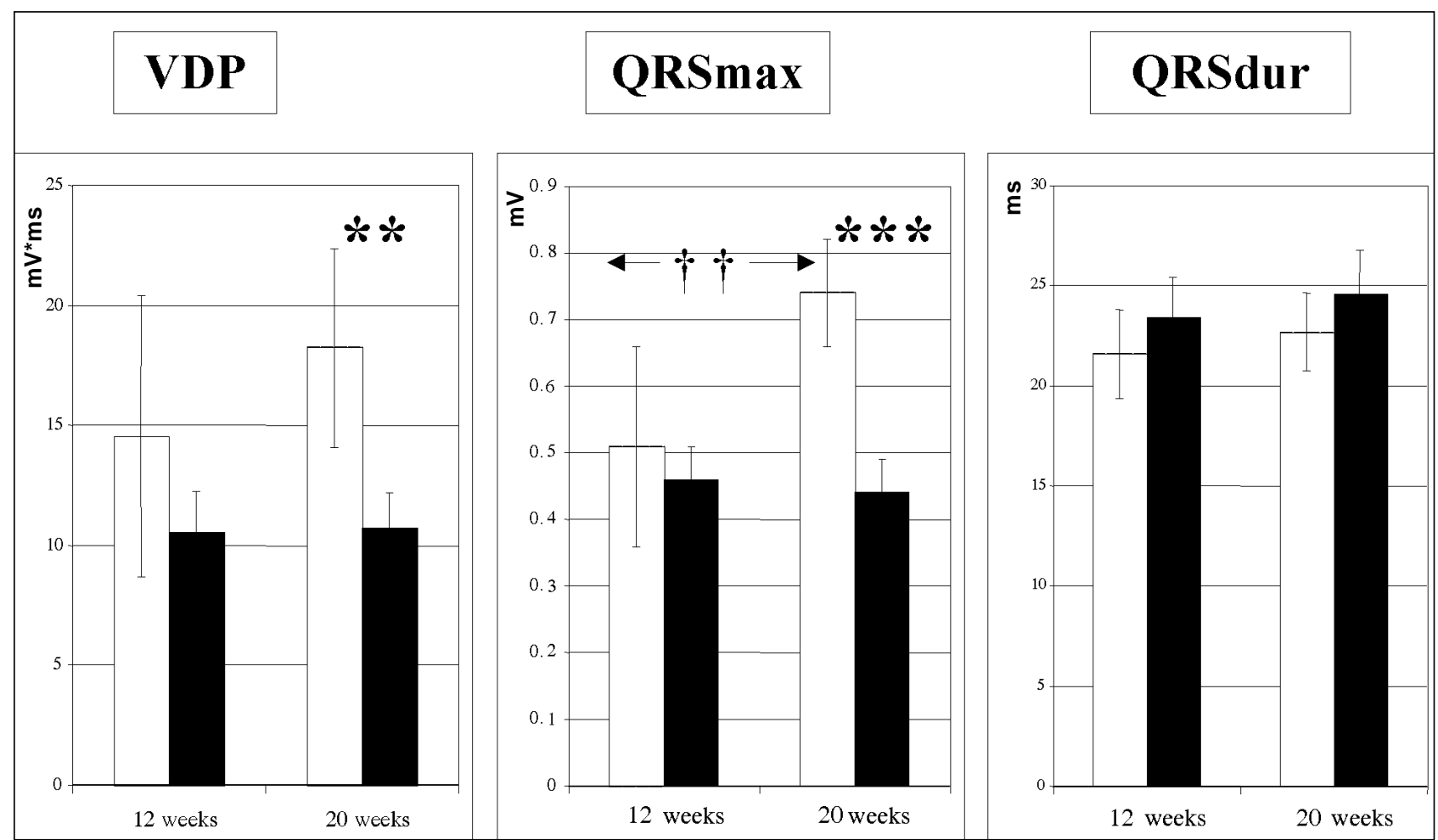

Fig. 1 - Changes in voltage-duration product (VDP), maximum spatial QRS vector magnitude (QRSmax), and QRS duration (QRSdur) in normotensive WKY rats (stripped columns) and spontaneously hypertensive rats (black columns) at the age of 12 and 20 weeks. Average values \pm SD are presented. Statistically significant difference WKY vs SHR: $* *=\mathrm{p}<0.01, * * *=\mathrm{p}<0.001 ;$ statistically significant difference WKY 12 weeks vs WKY 20 weeks: $\dagger \dagger=\mathrm{p}<0.01$.

tically significant at the age of 20 weeks $(10.73 \mathrm{mVms}$ in SHR compared with $18.23 \mathrm{mVms}$ in WKY, $<<0.01)$. The correlations between VDP and LVM and/or sBP were not significant in either WKY or in SHR.

QRSmax in SHR did not follow either the increase in sBP, LVM, or LVM/BW. The QRSmax values in SHR did not differ from those of WKY at the age of 12 weeks $(0.59 \pm 0.14$ $\mathrm{mV}$ versus $0.46 \pm 0.05 \mathrm{mV}$ ), and they were even lower in SHR at the age of 20 weeks $(0.74 \pm 0.08 \mathrm{mV}$ versus $0.44 \pm 0.05 \mathrm{mV}$, $\mathrm{p}<0.001)$. QRSmax correlated significantly with LVM only in WKY $(\mathrm{r}=0.6082, \mathrm{p}<0.05)$, but they did not correlate in SHR $(r=-0.1135)$. The correlation between QRSmax and SBP was not significant either in WKY or in SHR.

The QRS duration tended to be higher in SHR and increased with increasing age. However, this increase was not statistically significant. The correlations between QRSd and LVM and/or sBP were not significant either in WKY or in SHR.

\section{Discussion}

The main findings of this study were incongruent relations between the changes in LVM and VDP in SHR and WKY at the age of 12 and 20 weeks, namely the increase in VDP in WKY without changes in LVM, and lower values of VDP in SHR in spite of the significant increase in sBP, LVM, and LVM/BW. These findings were predominantly influenced by the QRSmax changes.
QRS voltage and left ventricular hypertrophy - A significant increase in QRSmax of about $45 \%$ in normotensive WKY rats was found during the follow-up period, while neither the LVM nor the LVM/BW were changed significantly. In rats, the period from 12 to 20 weeks refers to the period of adolescence and early adulthood. An LVM-independent increase in QRS amplitude in the period of adolescence and early adulthood has been documented both in experimental and clinical studies ${ }^{11-16}$. The mechanism of this increase in QRS voltage in healthy normotensive adolescents and young adults is not clear. Because WKY are genetically homogenous and do not develop hypertension, this increase in QRS voltage cannot be considered an early premorbid sign of hypertension potentially developing in later adulthood. The increase in QRSmax was not associated with parameters under study characterizing hypertrophy (sBP, LVM, LVM/BW); therefore, it cannot be attributed to the increase in LVM or to the progression of hypertrophy. However, we had to consider this increase while interpreting the comparison with age-matched SHR the more that this increase was in direct contrast to the QRS changes found in SHR in this study.

By contrast, the QRSmax in SHR did not change during the follow-up period, in spite of the significant progression of hypertension and left ventricular hypertrophy characterized by the significant increase in sBP, LVM, and the LVM/BW ratio. Additionally, the QRSmax values in SHR were significantly lower compared to with those in the age-matched WKY rats, and this difference was 
enhanced in the 20-week-old SHRs compared with that in the 12-week-old SHRs.

The finding of no significant change in QRSmax in SHR is of particular interest, because, according to the classical hypothesis, an increased QRS voltage should be expected as a consequence of the "enhanced electrical dominance" of the increased mass of the left ventricle. The voltage criteria are considered the most specific clinical findings for ECG diagnostics of LVH. In experimental studies, however, no consistency exists in the manner in which QRS amplitude has been affected by induced hypertrophy. Some studies have found the QRS voltage to be significantly increased ${ }^{17-19}$, others have found a nonsignificant trend of increase in QRS amplitude ${ }^{20}$, whereas some others have found the amplitude to be decreased in SHR compared with that in normotensive rats ${ }^{21-23}$. The high number of so-called false negative ECG results and low sensitivity of ECG voltage criteria in clinical diagnostics is also well documented ${ }^{24-27}$.

In our previous works ${ }^{28-30}$, we introduced the term "relative voltage deficit" to assign ECG findings where QRS voltage is lower than expected according to the increase in LVM in LVH. We hypothesized that a relative voltage deficit is conditioned by changes in electrogenesis in left ventricular hypertrophy.

In the present study, the rats were examined at the ages of 12 and 20 weeks. At this age in SHR, the heart is known to be appreciably hypertrophied and heart failure can be practically excluded, because it does not occur until about 18 months of age ${ }^{8,31}$. However, also at this age of animals and in the relatively early period of hypertension and $\mathrm{LVH}$, we observed a relative voltage deficit in SHR. We assume that it reflects the changes in active and passive electrical properties of myocardium during pathological hypertrophic growth of the left ventricle.

Additionally in this study, we found no significant correlation between QRSmax and LVM in SHR in contrast to the significant correlation between QRSmax and LVM in normotensive WKY rats. Similar results, ie, no significant correlation between QRS amplitude and LVM in SHR, were reported even in studies where a significant increase in the QRS amplitude in SHR was observed. Yamori et al ${ }^{17}$ have shown that the magnitude of the maximum spatial QRS vector was significantly related to blood pressure, but not to histometrical findings, such as heart weight, left ventricular weight, or the thickening of the left ventricular wall. Similarly, Snoeck et al ${ }^{18}$ found no significant correlation between QRS max and heart weight and QRSmax and left ventricular thicknes. It follows that the mass of the hypertrophied left ventricle has not been the major determinant of the recorded QRS amplitude, that the increased mass of the hypertrophied left ventricle has not resulted in the "electrical dominance" of the left ventricle and consequently in increased QRS amplitude.

Increased QTS voltage in LVH is theoretically attributed to the increased size of the electrical activation boundary according to the solid angle theory ${ }^{32}$. However, the solid angle theory considers not only the spatial determinants (the size of the solid angle depending on the size of the activation boundary), but the nonspatial determinants as well: transmembrane voltage differences and conductivity. It seems that the changes in nonspatial factors could be those additional factors, which could counterbalance the influence of the size of LV on the resultant $\mathrm{QRS}$ voltage and contribute to the relative voltage deficit.

QRS duration and left ventricular hypertrophy - In this study, the QRSd values in SHR tended to be higher compared with those in WKY, but this difference was not statistically significant, and the QRSd values both in WKY and SHR were higher at the $20^{\text {th }}$ week compared with those at the $12^{\text {th }}$ week, but the difference was not statistically significant.

The published data on the association between QRS duration and LVH in SHR are not consistent. Yamori et al ${ }^{17}$ found a significant prolongation in QRS duration in strokeprone SHR compared with that in WKY at the age of 18-24.5 weeks. Snoeck et al ${ }^{18}$ observed a prolongation in QRS duration in SHR. At 3 months (12 weeks), a slight but significant increase was already observed, and at 12 months ( 84 weeks), a significant difference was noticed. Similar results, ie, increase in QRS duration with age in SHR were reported by Mueller-Peedinghaus et $\mathrm{al}^{33}$. Ohtaka ${ }^{20}$ reported significantly prolonged QRS duration in SHR compared with that in WKY at the age of 5 months ( 20 weeks). Dunn at al ${ }^{34}$ also reported progressively increased QRS duration with progressive left ventricular hypertrophy in SHR. However, this increase was statistically significant only in the oldest age group $>76$ weeks. In younger age groups $(8-12,26-51,51-76$ weeks), the difference between SHR and WKY was not statistically significant. On the other hand, Hodgkin et $\mathrm{al}^{21} \mathrm{did}$ not find differences in the QRS duration between SHR and WKY at the age of 9-14 weeks.

In humans, the prolongation of QRS complex duration is regularly listed among electrocardiographic signs of LVH. QRS duration has also been found to correlate with left ventricular mass ${ }^{35-37}$. However, the prolonged QRS complex is not a specific finding for $\mathrm{LVH}$ and is considered supporting evidence in the presence of increased voltage ${ }^{38}$.

Changes in QRS duration in LVH may be attributed to the increased muscle mass itself, ie, to the longer time required to activate the increased mass of myocardium ${ }^{39}$. Additionally, changes in active and passive electrical properties of myocardium in LVH have been reported, such as conduction velocity, intracellular resistivity, gap junction organization, resistance, and the content of connexin $43^{40-48}$. It can be suggested that these changes are involved in the changes in QRS duration in LVH. These changes are extensively studied mainly in relation to their role in arrhythmogenesis. However, in terms of the solid angle theory, we can speculate that they can also be involved in the voltage changes in the QRS complex and counterbalance the influence of the increased mass.

In this study, the nonsignificant increase in QRSdur did not significantly influence the VDP and did not balance the opposite changes of $Q R S$ voltage.

Voltage-duration product and LVH - The findings of 
this study are in direct contrast with findings of clinical studies that report good performance of VDP in the detection of $\mathrm{LVH}^{2-7}$. In this study, the voltage-duration product in SHR did not reflect the increase in LVM at the age of 12 to 20 weeks. On the contrary, we observed a significant increase in VDP in control normotensive WKY rats without changes inLVM.

The difference in findings can be partly attributed to differences in the study designs and patient selection between our study and published reports from other clinical studies. In our study, the hypertrophy was studied in spontaneously hypertensive rats, ie, in a diagnostically well-defined group of experimental animals. The experiment was controlled for age, sex, and therapy. LVH was defined by the increase in sBP, LVM, and the LVM/BW ratio. In the clinical studies, the discriminative parameter between control and LVH groups was the only parameter, the increased LVM index. In the study of Molloy et $\mathrm{al}^{2}$, both control and LVH groups contained a variety of cardiovascular pathology. In another study ${ }^{3}$, the normal control group included also an unknown proportion of patients with mild hypertension, and on the other hand, the LVH group contained an unknown proportion of "normotensive patients," healthy subjects perhaps? The clinical studies were not controlled for age, sex, and therapy.

The included variety of pathology and the absence of the control for age, sex, and therapy implies that authors have not considered the changes of active and passive electrical properties in terms in the solid angle theory, namely the nonspatial determinants influencing the voltage of recorded electrocardiogram.

This is also reflected in the inconsistency in the application of the solid angle theory when discussing their result. In the case of the QRS voltage, the solid angle theory is used for argumentation, but only the spatial determinants and the increased QRS voltage is attributed to the increased size of the electrical activation boundary, ie, to the spatial determinants. However, this is not a complete citation, because the part of the nonspatial determinants is not mentioned. However, next, in the case of QRS prolongation, evidence for changed active and passive electrical properties is quoted. Paradoxically, these changes in conductivity are not considered in terms of the nonspatial determinants of the recorded voltage according to the solid angle theory.
In our previous works ${ }^{28-30}$, we repeatedly stressed the importance of nonspatial determinants of QRS voltage in LVH. We introduced the term "relative voltage deficit" for so-called false negative ECG results to stress that the enlarged LV in LVH are not strong generators of a cardio-electric field as is expected according to their mass. And we introduced a parameter for the quantification of the relative voltage deficit - the specific potential of the myocardium.

Limitations of the study - The present study has certain limitations inherent to the model of SHR used. Another limitation is the small number of experimental animals; however, the changes were consistent across groups.

Conclusion - In this study, the voltage-duration product did not reflect the increase in LVM in SHR at the age of 12 to 20 weeks, and, on the other hand, the increase in VDP in normotensive control WKY was not associated with the increase in LVM. In other words, the LVM was not the major determinant of the QRS voltage and consequently VDP. These findings focus attention on to the importance of the nonspatial determinants of the recorded QRS voltage in terms of the solid angle theory.

The clinical implications aim at the re-evaluation of the role of ECG in LVH diagnostics, especially in the case of the so-called false negative results and at the differentiation between the anatomical size of the heart as the source of the cardiac electric field and its electrogenetic properties. The alternative explanation for the so-called false negative ECG results in LVH is the relative voltage deficit, related to changes in active and passive electrical properties of the hypertrophied myocardium. The term relative voltage deficit refers to discrepancies in actual cases as indicating deviations from the "ideal" state. The changes in the relative QRS voltage in different stages of LVH should also be taken into account. A potential exists for studying the relative voltage deficit and its diagnostic and prognostic usefulness in the frame of diagnostics of LVH, of diffuse changes in the myocardium, in cardiovascular risk assessment, and for the evaluation of the effects of therapy.

\section{Acknowledgements}

This study was supported, in part, by the grant 1/ 7189/20 from The Science Grant Agency (VEGA), Slovak Republic.

\section{References}

1. Kannel WB. Epidemiology of cardiac hypertrophy. In: Sheridan DJ, editor. Left ventricular hypertrophy. London: Churchill Livingstone, 1998: 1-10.

2. Molloy TJ, Okin PM, Deverex RB, Kligfield P. Electrocardiographic detection of left ventricular hypertrophy by the simple QRS voltage-duration product. J Am Coll Cardiol 1992; 20: 1180-6.

3. Okin PM, Roman MJ, Devereux RB, Kligfield P. Electrocardiographic identification of increased left ventricular mass by simple voltage-duration products. J Am Coll Cardiol 25; 1995: 417-23.

4. Okin PM, Roman MJ, Devereux RB, Kligfield P. Electrocardiographic identifi- cation of left ventricular hypertrophy: test performance in relation to definition of hypertrophy and presence of obesity. J Am Coll Cardiol 1996; 27: 124-31.

5. Okin PM, Jern S, Devereux RB, Kjeldsen SE, Dahloef B, fot the LIFE Study Group. Effect of obesity on electrocardiographic left ventricular hypertrophy in hypertensive patients: the Losartan Intervention For Endpoint (LIFE) Reduction in Hypertension Study. Hypertension 2000; 35: 13-8.

6. Okin PM, Roman MJ, Devereux RB, Kligfield P. ECG identification of left ventricular hypertrophy. Relationship of test performance to body habitus. J Electrocardiol 1996; 29(suppl): 256-61. 
7. Norman JE Jr, Levy D. Adjustment of ECG left ventricular hypertrophy criteria for body mass index and age improves classification accuracy: the effect of hypertension and obesity. J Electrocardiol 1996; 29(suppl): 241-7.

8. Doggrell S, Brown L. Rats model of hypertension, cardiac hypertrophy and failure. Cardiovasc Res 1998; 39: 89-105.

9. Pfeffer MA, Frolich ED. Hemodynamic and myocardial function in young and old normotensive rats. Circ Res 1973; 32-33: 1-28.

10. Lindpaintner K, Kreutz R, Ganten D. Genetic variation in hypertensive and 'control' strains: what are we controlling for anyway? Hypertension 1992; 19: 428-30.

11. Osborne BE. The electrocardiogram of the rat. In: Budden R, Detweiler DK, Zbinden G, editors. The Rat Electrocardiogram in Pharmacology and Toxicology. Oxford: Pergamon Press, 1981: 15-27.

12. Simonson E. Differentiation between normal and abnormal in electrocardiography. St. Louis: C.V. Mosby Co, 1961: 86.

13. Macfarlane PW, Edenbrandt L, Pahlm O. 12-Lead Vectorcardiography. Oxford: Butterworth-Heinemann, 1995: 54.

14. Bayes de Luna A. Clinical electrocardiography. Mount Kisco, NY: Futura Publ. Co., 1993: 102

15. Bernadic M, Hulin I, Filipova S, Popperova E, Michalik D. Posturalne zmeny v ortogonalnom elektrokardiograme u zdravych adolescentov (Postural changes in the orthogonal electrocardiogram in healthy adolescents). Bratisl Lek Listy 1981; 76: 423-41. (in Slovak, summary in English).

16. Filipova S, Hulin I, Bernadic M. ECG mapping of the developmental changes of the ventricular activation in puberty and adolescence. In: Ruttkay-Nedecky I, Macfarlane PW, editors. Electrocardiology '83. Amsterdam: Excerpta Medica, 1984: 68-71

17. Yamori YM, Nara Y. Vectorcardiographic study of left ventricular hypertrophy in spontaneously hypertensive rats. Jpn Circ J 1976; 40: 1315-29.

18. Snoeck J, Wittesaele W, Vanagt E, Vrints C. Evolution of left ventricular hypertrophy and orthogonal - vectorcardiographic parameters in SHR. Jpn Heart J 1982; 23(Suppl): 477-9.

19. Tsutsumi T, Sekiya S, Osada H, Harumi K, Miyazawa T, Sato S. Vectorcardiogram with McFee-Parungao lead system in spontaneously hypertensive rats. Jpn Circ J 1985; 49: 1159-66.

20. Ohtaka M. Vectorcardiographical and pathological approach to the relationship between cardiac hypertrophy and coronary arteriosclerosis in spontaneously hypertensive rats (SHR). Jpn Circ J 1980; 44: 283-93.

21. Hodgkin BC, Nelson CV, Angelakos ET. Cardiac electrical resultant dipole moment of spontaneously hypertensive rats. Am J Physiol 1981; 241 (Heart Circ Physiol 10): H541- H6.

22. Roschevsky MP, Barabanova VV, Gagiev NG, et al. Experimental investigation of cardiac electric filed. In: Schubert E, editor. Electrocardiology '87. Berlin: Akademie-Verlag, 1988: 229-31.

23. Roschevsky MP, Barabanova VV, Gagiev NG, et al. Electric field of the rat heart in spontaneous hypertension. Sechenov Physiological Journal of the USSR 1988; 74: 1140-8, (in Russian, summary in English).

24. Romhilt DW, Bove KE, Norris RJ, et al. A critical appraisal of the electrocardiographic criteria for the diagnosis of left ventricular hypertrophy. Circulation 1969; 40: 185-95.

25. Reichek N, Devereux RB. Left ventricular hypertrophy: relationship of anatomic, echocardiographic and electrocardiographic findings. Circulation 1981; 63: 1391-8.

26. Casale PN, Devereux RB, Kligfield P, et al. Electrocardiographic detection of left ventricular hypertrophy: development and prospective validation of improved criteria. J Am Coll Cardiol 1985; 6: 572-80.

27. Kimura M, Matsushita S, Nakahara K-I, Myakawa A, Kuramoto K. Evaluation of electrocardiographic criteria for left ventricular hypertrophy based on anatomical comparison. J Electrocardiol 1987; 20: 369-74

28. Bacharova L, Melotova J, Sedlakova K. The "specific potential" as a parameter of myocardial changes in left ventricular hypertrophy. In: Abel H, editor. Electro- cardiology 1988. Amsterdam: Elsevier Science Publisher, Excerpta Medica 1989: 195-8.

29. Bacharova L. Reasoning for introducing a new parameter for assessment of myocardial status - the specific potential of myocardium. In: Cohen ME, Hudson DL, editors. Comparative Approaches to Medical Reasoning. Singapore: World Scientific Publ Co., 1995: 217-41.

30. Bacharova L. Effect of left ventricular hypertrophy on the cardiac electrical field: The concept of the specific potential of myocardium. Exp Clin Cardiol 1998; 3: 128-33.

31. Brooksby P, Levi AJ, Jones JV. The electrophysiological characteristics of hypertrophied ventricular myocytes from the spontaneously hypertensive rat. J Hypertension 1993; 11: 611-22.

32. Holland RP, Arnsdorf MF. Solid angle theory and the electrocardiogram" physiologic and quantitative interpretation. Prog Cardiovasc Dis 1977; 19: 431- 57.

33. Mueller-Peedinghaus R, Kuehl UG, Buschmann G. Comparison of ECG and morphological parameters in male and female spontaneously hypertensive rats (SHR). In: Budden R, Detweiler DK, Zbinden G, editors. The Rat Electrocardiogram in Pharmacology and Toxicology. Oxford: Pergamon Press, 1981: 145-54.

34. DunnFG,Pfeffer MA, Frolich ED.ECG alterations with progressive left ventricular hypertrophy in spontaneous hypertension. Clin Exp Hypertension 1978; 1: 67-86.

35. Vine DL, Finchum RN, Dodge HT, Bancroft WH Jr, Hurst DC. Comparison of the vectorcardiogram with the electrocardiogram in the prediction of left ventricular size. Circulation 1971; 43: 547-58.

36. Holt JH Jr, Barnard ACL, Lynn MS. A study of the human heart as a multiple dipole electrical source. II. Diagnosis and quantification of left ventricular hypertrophy. Circulation 1969; 40: 697-710.

37. Dunn RA, Pipberger HV, Holt JH Jr, Barnard ACL, Pipberger HA. Performance of conventional orthogonal and multiple-dipole electrocardiograms in estimating left ventricular muscle mass. Circulation 1979; 60: 1350-3.

38. Chou T-Ch. Electrocardiography in clinical practice. Adult and pediatric. $4^{\text {th }}$ ed Philadelphia: W.B. Saunders Co., 1996: 40.

39. Uhley HN, Proctor J. Study of the transmembrane action potential, electrogram, electrocardiogram and vectorcardiogram of rats with left ventricular hypertrophy. Am J Cardiol 1961; 7: 211-7.

40. Fry CH, Carey P, Mundy AR, Sheridan DJ. Altered conduction in left ventricular hypertrophy. In: Sheridan J, editor. Left ventricular hypertrophy. London: Churchill Livingstone, 1998: 113-20.

41. Toyoshima H, Park Y-D, Ishikawa, et al. Effect of ventricular hypertrophy on conduction velocity of activation front in the ventricular myocardium. Am J Cardiol 1982; 49: 1938-45.

42. Winterton SJ, Turner MA, O'Gorman DJ, Flores NA, Sheridan DJ. Hypertrophy causes delayed conduction in human and guinea pig myocardium: accentuation during ischaemic perfusion. Cardiovasc Res 1994; 23: 47-54

43. Cooklin M, Wallis WRJ, Sheridan DJ, Fry CH. Changes in cell-to-cell electrical coupling associated with left ventricular hypertrophy. Circ Res 1997; 80: 765-71.

44. McIntyre H, Fry CH. Abnormal action potential propagation in isolated human hypertrophied left ventricular myocardium. J Cardiovasc Electrophysiol 1997; 8: 887-94.

45. Carey PA, Cooklin M, Sheridan DJ, Fry CH. Estimation of gap-junction resistance in hypertrophied and hypoxic guinea pig myocardium. J Physiol 1997; 504: 96-7P

46. Peters NS, Green CR, Poole-Wilson PA, Severs NJ. Reduced content of connexin43 gap junctions in ventricular myocardium from hypertrophied and ischaemic human hearts. Circulation 1993; 88: 864-75.

47. Goldfine SM, Walcott B, Brink PR, Magid NM, Borer JS. Myocardial connexin43 expression in left ventricular hypertrophy resulting from aortic regurgitation. Cardiovasc Pathol 1999; 8: 1-6.

48. Yamamoto S, James TN, Sawada K-I, Okabe M, Kawamura K. Generation of new intercellular junctions between cardiocytes. A possible mechanism compensating for mechanical overload in the hypertrophied human adult myocardium. Circ Res 1996; 78: 362-70 